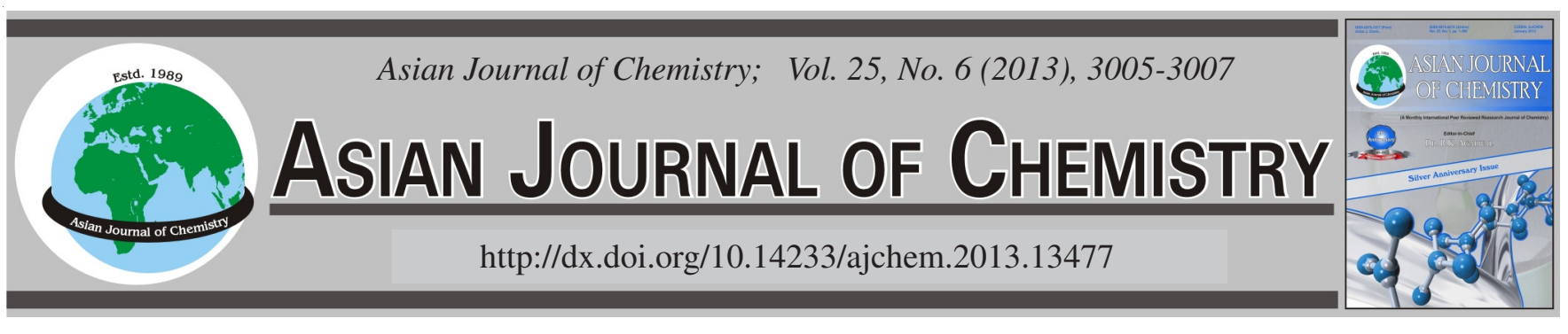

\title{
Thermal Property of Poly(L-lactic Acid)/Sodium Alginate Composites: Crystallization and Melting Behaviour
}

\author{
YAN-HuA CAI
}

School of Materials and Chemical Engineering, Chongqing University of Arts and Sciences, Yongchuan, Chongqing 402160, P.R. China

Corresponding author: E-mail: caiyh651@yahoo.com.cn

\begin{abstract}
The crystallization and melting behaviour of poly(L-lactic acid) (PLLA)/sodium alginate (SA) composites with different content of sodium alginate has been investigated by differential scanning calorimetry and depolarized-light intensity measurement. Sodium alginate is effective nucleating agent for the crystallization and can increase the overall crystallization rate of PLLA. With the addition of $3 \%$ sodium alginate, the induction time and crystallization half-time of PLLA/SA composites decrease from 12.8-0.7 and 26.5-1.4 min at 105 ${ }^{\circ} \mathrm{C}$, respectively. Sodium alginate addition makes crystallization peak for PLLA become sharp in the cooling process. The melting behaviour of PLLA $3 \%$ SA composites during non-isothermal crystallization at different cooling rate shows that cooling rate of non-isothermal crystallization process obviously affect the melting behaviour of PLLA, with increasing of cooling rate, the double melting peak degenerates to single melting peak.
\end{abstract}

Key Words: Poly(L-lactic acid), Crystallization, Sodium alginate, Nucleating effect.

\section{INTRODUCTION}

Poly(L-lactic acid) (PLLA) is important environmentally friendly polymer and is widely used as an alternative material to petro-derived polymers due to the producibility from renewable resources. Poly(L-lactic acid) is expected as carbon neutral material from the ecological point of view. However, until now, PLLA still has some disadvantages that restrict its practical applications, in particular, slow crystallization rate, low crystalline degree and poor heat resistance for practical use compared to the conventional thermoplastic. Thus, the study on the crystallization and melting behaviour of PLLA is of great important not only from academic interest but also from the engineering viewpoint. In fact, thermal property of PLLA and its composites have been reported by a number of researchers and the most viable method to increase the overall crystallization rate is the blending with nucleating agent. Layered silicate as nucleating agent of PLLA is earliest used to increase crystallization rate of PLLA. In the family of layered silicate, talc, montmorillonite, layered metal phosphonates are widely used ${ }^{1,2}$. In addition of silica ${ }^{3}$, carbon nanotube ${ }^{4}$, etc., are widely used to improve crystallization of PLLA as a nucleating agent. To further develop more nucleating agents of PLLA, in this paper, sodium alginate (SA) is used to be nucleating agent of PLLA and the crystallization and melting behaviour of PLLA/SA composites were investigated by differential scanning calorimetry and depolarized-light intensity measurement.

\section{EXPERIMENTAL}

Poly(L-lactic acid) (2002D) was purchased from Nature Works LLC, USA. The sodium alginate was procured from Chengdu Kelong Chemical Reagents Company.

Preparation of PLLA/sodium alginate composites: Blending of PLLA and sodium alginate was performed on a counter-rotating mixer and the preparation process of PLLA/ SA composites is similar to that of our previous paper ${ }^{5}$.

Differential scanning calorimeter (DSC): The nonisothermal crystallization behaviour of PLLA/SA composites was measured by DSC Q2000 (TA Instrumrnts-Waters LLC, USA). The temperature and heat flow at different heating rate were calibrated using an indium standard. The sample was heated to $190^{\circ} \mathrm{C}$ and maintained at that temperature for $5 \mathrm{~min}$ to make sure that the polymer crystals were melted completely. Then the samples were cooled from the melt state to $20^{\circ} \mathrm{C}$ at cooling rate of $1{ }^{\circ} \mathrm{C} / \mathrm{min}$. The melting behaviour was also measured by DSC Q2000 instrument.

Depolarized-light intensity measurement: The overall isothermal crystallization behaviour of PLLA was investigated by GJY-1lloptical depolarizer in the region from $100-120^{\circ} \mathrm{C}$. The electronic signals transformed from the measured optical 
depolarizer were amplified and then recorded on the recording and data acquisition for further analyzing.

\section{RESULTS AND DISCUSSION}

Overall isothermal crystallization of PLLA/SA compo-

sites: Because the crystallization rate of sodium alginatecontaining PLLA samples is very fast and there existes some time from melt to the desired crystallization temperature upon rapid cooling rate, thus, isothermal crystallization behaviour is investigated by using depolarized-light intensity technique ${ }^{6}$ as the transmitted light intensity (I) increases with the increasing of crystallinity and finally levels off when crystallization completes. In present study, we used the relative light intensity $\left(\mathrm{I}_{\mathrm{r}}\right)$ defined by the following equation as an index of crystallinity:

$$
\mathrm{I}_{\mathrm{r}}(\%)=100 \times \frac{\left(\mathrm{I}_{\mathrm{r}}-\mathrm{I}_{0}\right)}{\left(\mathrm{I}_{\infty}-\mathrm{I}_{0}\right)}
$$

where $I_{t}$ and $I_{0}$ are the I values at $t_{c}=t$ and 0 , respectively, $I_{\infty}$ is the I value when crystallization completes. Figs. 1 and 2 shows the effect of the SA contents and the crystallization temperature against the induction time $\left(\mathrm{t}_{\mathrm{i}}\right)$ and half time of overall PLLA crystallization $\left(t_{1 / 2}\right)$. Firstly, with the increasing of crystallization temperature, the $t_{i}$ and $t_{1 / 2}$ becomes shorter and possesses a minimum value at $105^{\circ} \mathrm{C}$, resulting from the enhancement of the macromolecule segment active ability with the increasing of crystallization temperature. However, it is hard for PLLA to form the spherulite if the $T_{c}$ is too high because the excessive macromolecule segment activity restricts the formation of spherulite and the $t_{i}$ and $t_{1 / 2}$ become longer with further increasing of crystallization temperature. On the other hand, the $t_{i}$ of PLLA/SA composites has minimum value $1.4 \mathrm{~min}$ with $1 \% \mathrm{SA}$. And the ti become longer, when SA content is further increased. However, the trend of $\mathrm{t}_{1 / 2}$ with $\mathrm{SA}$ content is not similar to that of $t_{i}$. Increasing of SA makes $t_{1 / 2}$ decrease. To compare with the neat PLLA, the $\mathrm{t}_{1 / 2}$ of PLLA/SA composites decreased from 26.5-1.4 min at $105{ }^{\circ} \mathrm{C}$, with $3 \% \mathrm{SA}$ contents at $105^{\circ} \mathrm{C}$. Addition of SA makes $\mathrm{t}_{\mathrm{i}}$ and $\mathrm{t}_{1 / 2}$ of PLLA/ SA composites highly decreases, which indicate that SA as it plays an excellent heterogeneous nucleation role.

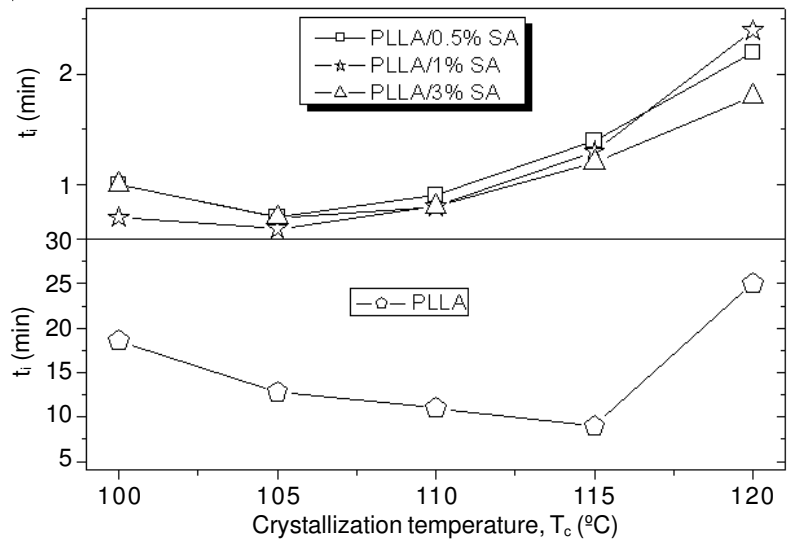

Fig. 1. The induction time of overall crystallization of PLLA and PLLA/ SA composites with the crystallization temperature $\left(T_{c}\right)$

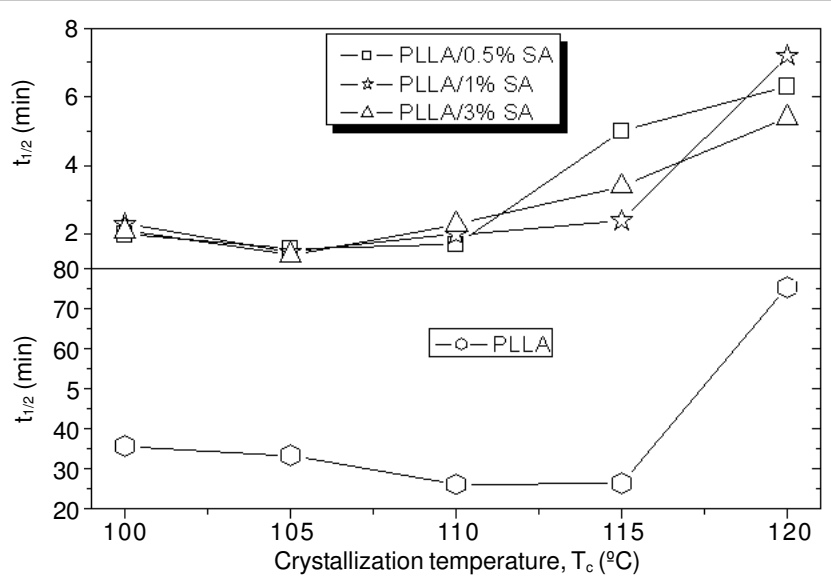

Fig. 2. The half-time of overall crystallization of PLLA and PLLA/SA composites with the crystallization temperature $\left(\mathrm{T}_{\mathrm{c}}\right)$

Non-isothermal crystallization of PLLA/SA composites: It is very important to investigate non-isothermal crystallization to obtain the information useful for industrial applications of PLLA. Fig. 3 shows the DSC curves of nonisothermal crystallization of PLLA/SA composites from melt at a different cooling rate. As seen in Fig. 3, upon different cooling rate, the crystallization peak of PLLA can almost not be detected, which shows that the crystallization of neat PLLA is very slow. With addition of sodium alginate, crystallization peak appears in the DSC cooling curve. Compared to the neat PLLA, SA addition leads to the shift of crystallization peak to higher temperature indicating the increase of crystallization temperature. On the other hand, crystallization peak for PLLA containing sodium alginate becomes much sharper in the cooling process, this result shows that sodium alginate can serve as a nucleating agent for the crystallization and increase the overall crystallization rate of PLLA.

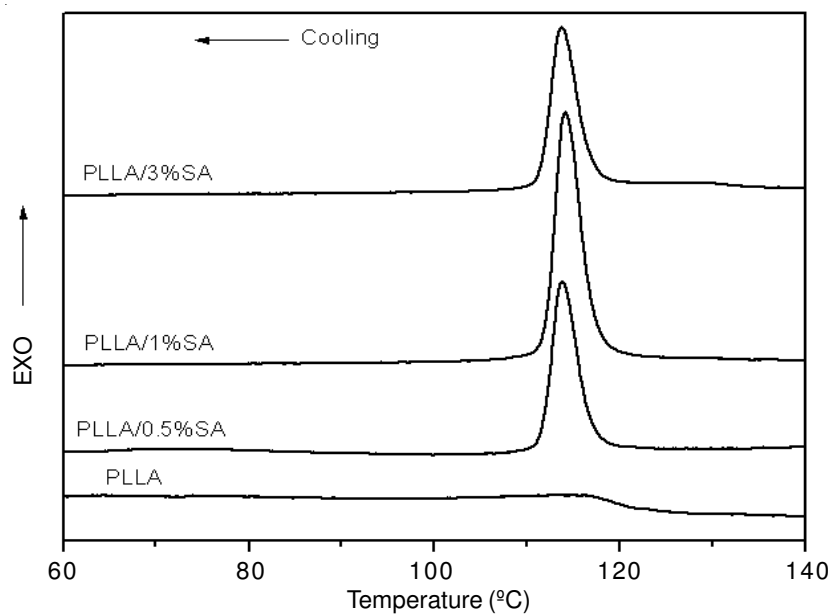

Fig. 3. DSC of PLLA and PLLA/SA composites crystallized from melt at a cooling of $1^{\circ} \mathrm{C} / \mathrm{min}$

Melting behaviour of PLLA/3 \%SA composites after non-isothermal crystallization at different cooling rate: Fig. 4 shows the melting behaviour of PLLA/3\%SA composites at a heating rate of $10^{\circ} \mathrm{C} / \mathrm{min}$ after non-isothermal crystallization at different cooling rate. With increasing of cooling rate, the double melting peak degenerates to single melting peak. It 


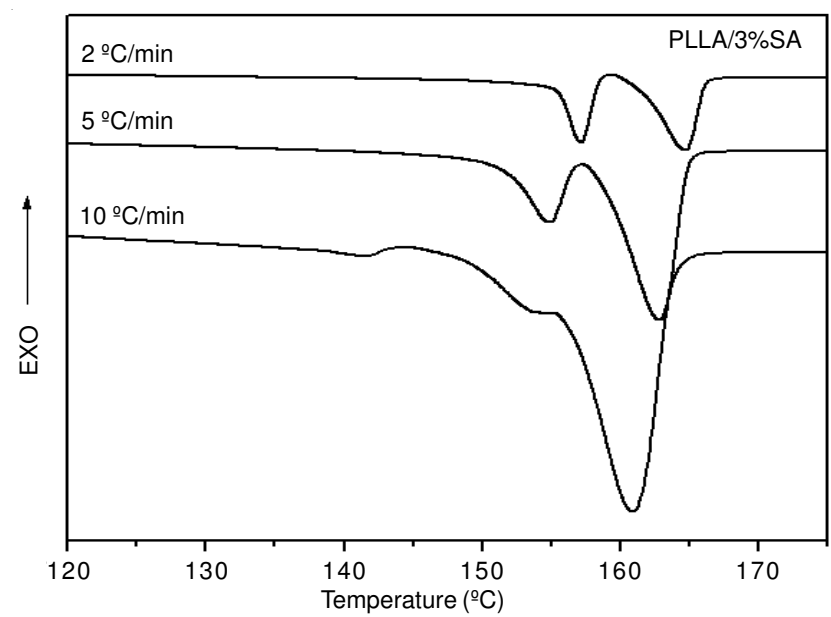

Fig. 4. Melting behaviour of PLLA/3\%SA composites at a different heating rate during non-isothermal crystallization at different cooling rate

is clear that the high-temperature and low-temperature melting peaks of PLLA/3\%SA composites shift to low-temperature with increasing of cooling rate, the reason is that the increasing of cooling rate makes the crystallization of PLLA to be incomplete and the prefect of spherulite becomes worse. Meantime, there exist obvious second crystallization process after non-isothermal crystallization and the second crystallization peak is more obvious with increasing of cooling rate, this is due to more incomplete of crystallization of PLLA with increasing of cooling rate. According to literature ${ }^{7}$, the doublemelting behaviour of PLLA assigns to melting-recrystallization. The low-temperature melting peak is attributed to the primary crystallites formed during non-isothermal crystallization process and high-temperature melting peak reflect the relatively prefect lamella stacks resulted from recrystallization during the heating scan.

\section{Conclusion}

The thermal behaviour of PLLA/SA composites were investigated by DSC and depolarized-light intensity measurement in detail. With the addition of $3 \%$ SA, the crystallization half-time of PLLA/SA composites decreased from 26.5-1.4 min at $105^{\circ} \mathrm{C}$. The melting behaviour of PLLA $3 \%$ SA composites during non-isothermal crystallization at different cooling rate showed that cooling rate of non-isothermal crystallization process obviously affect the melting behaviour of PLLA.

\section{ACKNOWLEDGEMENTS}

This work was supported by The Ministry of Science and Technology of P.R. China (Project No. 2007BAE42B00), Natural Science Foundation Project of CQ CSTC (Project No. cstc2012jjA50001), Postdoctoral Science Foundation Project of ChongQing (Project No. XM20120035) and ChongQing University of Arts and Sciences (Project No. R2012CH10, 2012PYXM04 and Z2011CL11).

\section{REFERENCES}

1. X.X. Li, J.B. Yin, Z.Y. Yu, S.F. Yan, X.C. Lu, Y.J. Wang, B. Cao and X.S. Chen, Polym. Comp., 30, 1338 (2009).

2. S.M. Huang, J.J. Hwang, H.J. Liu and L.H. Lin, J. Appl. Polym. Sci., 117, 434 (2010).

3. S.F. Yan, J.B. Yin, Y. Yang, Z.Z. Dai, J. Ma and X.S. Chen, Polymer, 48, 1688 (2007).

4. Y.Y. Zhao, Z.B. Qiu and W.T. Yang, Comp. Sci Technol., 69, 627 (2009).

5. Y.H. Cai, S.F. Yan, J.B. Yin, Y.Q. Fan and X.S. Chen, J. Appl. Polym. Sci., 121, 1408 (2011).

6. T. Miyata and T. Masuko, Polymer, 39, 5515 (1998).

7. M. Yasuniwa, S. Tsubakihara, Y. Sugimoto and C. Nakafuku, J. Polym. Sci. B: Polym. Phys., 42, 25 (2004). 